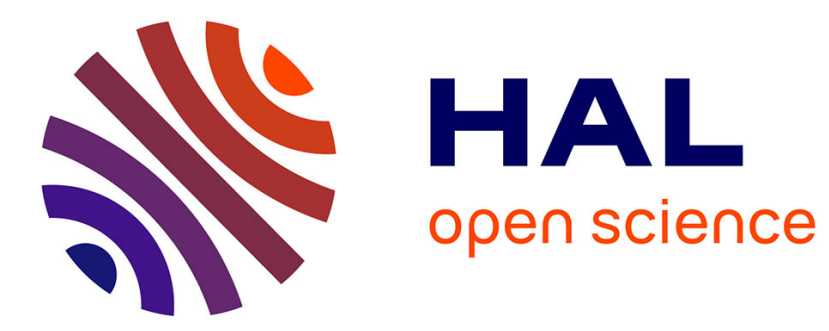

\title{
La gauche et la diversité: une occasion manquée, de nouveau
}

Vincent Tiberj

\section{To cite this version:}

Vincent Tiberj. La gauche et la diversité: une occasion manquée, de nouveau. Mouvements: des idées et des luttes, 2017, La gauche est morte, vive la gauche!, 89, pp.53-61. 10.3917/mouv.089.0053 . halshs-01528742

\section{HAL Id: halshs-01528742 \\ https://shs.hal.science/halshs-01528742}

Submitted on 29 May 2017

HAL is a multi-disciplinary open access archive for the deposit and dissemination of scientific research documents, whether they are published or not. The documents may come from teaching and research institutions in France or abroad, or from public or private research centers.
L'archive ouverte pluridisciplinaire HAL, est destinée au dépôt et à la diffusion de documents scientifiques de niveau recherche, publiés ou non, émanant des établissements d'enseignement et de recherche français ou étrangers, des laboratoires publics ou privés. 


\title{
LA GAUCHE ET LA DIVERSITÉ : UNE OCCASION MANQUÉE, DE NOUVEAU \\ Vincent Tiberj
}

\author{
La Découverte | «Mouvements »
}

2017/1 n 89 | pages 53 à 61

ISSN 1291-6412

ISBN 9782707194527

Article disponible en ligne à l'adresse :

http://www.cairn.info/revue-mouvements-2017-1-page-53.htm

\section{Pour citer cet article :}

Vincent Tiberj, «La gauche et la diversité : une occasion manquée, de nouveau », Mouvements 2017/1 ( $\mathrm{n}^{\circ} 89$ ), p. 53-61.

DOI 10.3917/mouv.089.0053

Distribution électronique Cairn.info pour La Découverte.

(C) La Découverte. Tous droits réservés pour tous pays.

La reproduction ou représentation de cet article, notamment par photocopie, n'est autorisée que dans les limites des conditions générales d'utilisation du site ou, le cas échéant, des conditions générales de la licence souscrite par votre établissement. Toute autre reproduction ou représentation, en tout ou partie, sous quelque forme et de quelque manière que ce soit, est interdite sauf accord préalable et écrit de l'éditeur, en dehors des cas prévus par la législation en vigueur en France. Il est précisé que son stockage dans une base de données est également interdit. 


\section{La gauche et la diversité : une occasion manquée, de nouveau}

\author{
La gauche continue de voir la diversité avec des œillères qui \\ l'empêche de répondre aux enjeux d'une société française \\ multiculturelle. L'intégration, au sens classique du terme, est faite. \\ L'opinion, si souvent invoquée, plus ouverte que les politiques, \\ en a pris acte. Les récents attentats terroristes n'ont pas inversé \\ la tendance. Vincent Tiberj explique que ce sont nos politiques \\ publiques qu'il faut changer, en sortant enfin d'un modèle \\ républicain daté et inégalitaire.
}

$\mathrm{L}^{\prime}$

immigration et l'intégration ont pris une importance toute particulière dans la France de l'après 2001. C'est même devenu un reconfigurations idéologiques de la droite, qui désormais est simultanément libérale économiquement et conservatrice culturellement ${ }^{1}$, à tel point que la manière dont ces sujets sont abordés semble désormais dictée par la droite, notamment autour du cadrage religieux et des dangers réels ou supposés que ferait courir l'islam à la France. Ce n'est pas un hasard si François Fillon, dans l'entre-deux-tours des primaires, a ainsi proclamé que la France n'est pas multiculturelle, alors que l'athéisme et le pluralisme religieux sont une réalité difficilement contestable, tout comme la multitude des modes de vie et des langues.

Face à cette donne, la gauche en général, et la gauche de gouvernement en particulier, est beaucoup plus divisée. Certains en sont encore à discuter de la nécessité de prendre en compte ou non les questions de diversité et de tolérance dans le programme de la gauche ou à suivre les cadrages et interprétations du fait musulman qui viennent pourtant du camp d'en face. Les attentats de janvier et novembre 2015 ne les ont pas amenés à sortir du cadrage sécuritaire de l'immigration. Pourtant, dans la coalition d'électeurs qui a porté François Hollande au pouvoir, les Français d'origine immigrée et les habitants des quartiers populaires ont été particulièrement présents.

Ce n'est pas tant ici la légitimité de s'emparer de ces questions qui constitue l'objet de cette contribution, mais plutôt la volonté de revenir

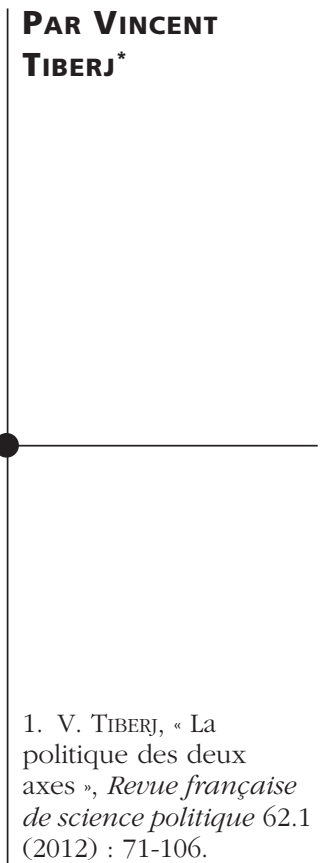

* Professeur des Universités associé, Sciences Po Bordeaux. 
2. Ce qu'une approche un peu trop monocausale laisserait à penser (H. LAGRANGE, Le déni des cultures, Paris, Seuil, 2010).

3. Voir C. Beauchemin, C. Hamel, P. Simon (éd.), "Enquête "Trajectoires et Origines " :

la diversité des populations en France ", Document de travail de l'Ined $n^{\circ} 168,2010$.

sur des prêts-à-penser qui empêchent la société et les élus politiques français de les comprendre pleinement et plus sereinement. C'est ainsi que nous avons décidé de revenir sur trois points qui nous paraissent essentiels pour éviter de voir la France de la diversité avec des oeillères. La notion même de "crise de l'intégration " doit être mieux comprise. L'électorat français n'est pas aussi xénophobe et anti-immigré qu'on le croit. Enfin et surtout, la France n'est plus un modèle d'intégration en Europe. Autant de résultats qui obligent collectivement à revoir certaines routines de pensée de la part des responsables politiques, notamment à gauche.

\section{- Une " crise de l'intégration " ?}

Quand on parle d'immigration, c'est bien trop souvent pour l'associer au chômage et à la misère sociale, à l'insécurité, ou encore au communautarisme et à l'intégrisme religieux. Les attentats n'ont fait qu'exacerber une culture de la suspicion qui était déjà très présente. Pourtant, dès lors qu'on change de focale, la France de la diversité est loin de correspondre au portrait catastrophique qui en est fait et les responsabilités des problèmes ne sont peut-être pas du côté des usual suspects.

En matière d'immigration, il est coutumier de pointer du doigt les comportements d'une infime partie d'une population pour juger de l'ensemble de cette population. Les Musulmans seraient ainsi tous représentés par les barbus, les Noirs auraient des tendances criminogènes ${ }^{2}$, etc. D'ailleurs, c'est bien connu, les trains qui arrivent en retard sont représentatifs de l'ensemble des trains qui circulent.

Echec scolaire? Dans l'enquête Trajectoire et origines $(\mathrm{TeO})^{3} \mathrm{de}$

\section{En matière d'immigration, il est coutumier de pointer du doigt les comportements d'une infime partie d'une population pour juger de l'ensemble de cette population.}

2008-2009, les deuxièmes générations originaires d'Afrique sahélienne sont $51,5 \%$ à avoir au moins un baccalauréat, parmi les originaires de l'Afrique centrale, d'Algérie, du Maroc ou de la Tunisie, ils sont respectivement $62,5 \%, 44,5 \%$ et $59 \%$. Certes, ils

\author{
4. Y. Brinbaum, \\ L. Moguerou, \\ J.L. PRIMON. "Les \\ enfants d'immigrés ont \\ des parcours scolaires \\ différenciés selon leur \\ origine migratoire ", \\ Insee Références \\ Immigrés et descendants \\ d'immigrés en France : \\ 43-49, 2012. \\ 5. S. Brouard, V.TiberJ, \\ Français comme les \\ autres?, Paris, presses \\ de Sciences Po, 2005.
}

sont moins diplômés que les deuxièmes générations originaires de l'Asie du Sud Est $(72,5 \%)$ mais largement plus que la population française dans son ensemble (37\%). Et encore ne tient-on pas compte du niveau social des parents dont on sait combien il pèse sur le destin scolaire. Dès lors qu'on tient compte de ces aspects, certains enfants d'immigrés réussissent même mieux que les enfants du reste de la population ${ }^{4}$.

Repli sur soi et communautarisme ? Avec Sylvain Brouard 5 , nous avons estimé à moins de $5 \%$ la proportion des Français d'origine maghrébine, africaine et turque rejetant l'identité française au profit d'une identité communautaire ou religieuse. On peut s'inquiéter de cette attitude minoritaire, mais cela implique que $95 \%$ de cette population ne sont pas concernés. 
Islamisation et remise en question des valeurs républicaines ? Oui l'islam est en progression en France et reste la religion des enfants de l'immigration maghrébine et africaine. Pourtant, être musulman n'induit pas de rejet de la laïcité : pour plus de $80 \%$ des musulmans, celle-ci évoque "quelque chose de positif " et une proportion similaire est d'accord avec l'idée qu'" en France, seule la laïcité permet à des gens de convictions différentes de vivre ensemble". Quand on analyse le rapport à la religion des Français nés dans les années 1980 et après, on compte certes $10 \%$ de musulmans déclarés et $12 \%$ de catholiques pratiquants, mais $53 \%$ se déclarent sans-religion. Autrement dit, l'islam est une réalité française (entre 3,9 et 4,2 millions de croyants ${ }^{6}$ ) mais elle est loin d'être la seule. La focale sur l'islam doit être relativisée, tant à droite qu'à gauche.

Pourquoi alors autant de signaux d'alarme? Difficile de nier les taux de chômage dramatiques dans les cités, difficile de nier certains des appels des musulmans les plus conservateurs, difficile de nier la difficulté parfois d'être jeune fille ou gay dans les cités. Mais il faut savoir faire la part des choses entre combattre des phénomènes qui doivent être traités politiquement et les amalgames hâtifs. C'est ce que permet la sociologie en allant par exemple au-delà du comportement de minorités, en permettant de redonner une voix à ces majorités silencieuses de la diversité.

C'est aussi un devoir politique. Beaucoup d'efforts répressifs ont été consacrés à la burqa : le ministère de l'Intérieur relève 1644 verbalisations en six ans d'application de son interdiction, ce qui montre bien la très faible proportion de femmes qui ont adopté cette pratique extrême. Cela n'a pourtant pas empêché Manuel Valls de prendre position sur la pratique du burkini dont on peut se douter qu'elle est encore plus marginale. Pourtant, dans le même temps, on ne tiendra pas compte de problèmes qui empoisonnent la vie quotidienne de milliers d'enfants d'immigrés. Parmi les deuxièmes générations originaires d'Afrique, du Maghreb et les Ultramarins installés en France - qui n'ont en commun avec les immigrés que la couleur de peau -, près de $50 \%$ d'entre eux déclarent avoir été victimes d'injures racistes dans leur vie, entre $20 \%$ et $27 \%$ ont été contrôlés plusieurs fois par la police lors de la dernière année, et entre $38 \%$ et $46 \%$ ont fait l'objet de comportement discriminatoire dans leurs rapports aux administrations et aux banques lors des cinq dernières années (source : enquête $\mathrm{TeO}$ ).

La crise de l'intégration, à qui la faute ? Bien sûr le président François Hollande a fait entendre à plusieurs reprises un discours d'ouverture, notamment lors de l'inauguration (très tardive) du musée de l'Histoire de l'Immigration en décembre 2014 ou pendant ses allocutions postattentats où il a déclaré lutter contre les amalgames. Pourtant, ce travail de
6. P. SimON, V. Tiberj. Sécularisation ou regain religieux : la religiosité des immigrés et de leurs descendants. Documents de travail de l'INED, No. 196. 2013. 
reconnaissance symbolique est difficilement audible dès lors que le même responsable va proposer juste après de mettre en place la déchéance de nationalité et défendre ce projet pendant plusieurs mois.

\section{- Une opinion anti-immigrée ?}

Derrière les nombreux discours sur l'immigration, l'insécurité ou l'intégration, on retrouve quasiment systématiquement l'argument de la volonté du peuple contre des élites incapables de comprendre les vrais problèmes du quotidien. Le quinquennat qui s'achève n'aura pas été très différent, avec les thèses défendues par des intellectuels de droite comme Alain Finkielkraut ou Eric Zemmour, mais aussi par des analystes plus proches de la gauche comme le politiste Laurent Bouvet et son concept d'insécurité culturelle ou le géographe Christophe Guilluy et son approche de la France périphérique. Autrement dit, les Français d'en bas seraient en demande de plus de répression face à l'immigration et aux jeunes des cités. D’ailleurs la montée du FN aux européennes de 2014 et aux départementales et régionales de 2015 serait le signe évident d'un climat tendu. Climat auquel la gauche réagit, mais est-ce de la bonne manière?

Figure 1 : L'indice longitudinal de tolérance (1990-2016)

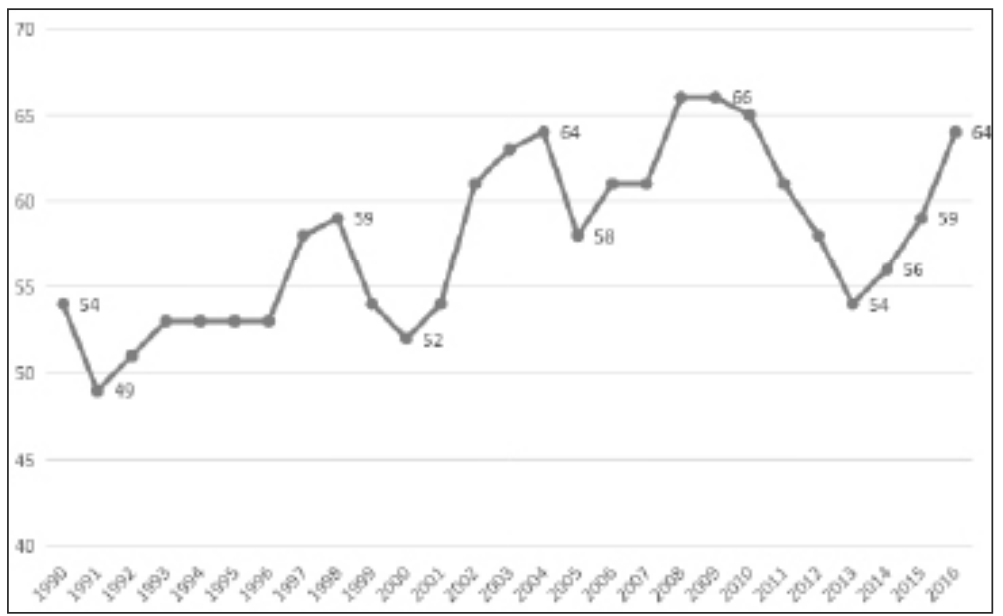

Calculs de Vincent Tiberj à partir du baromètre CNCDH. Lecture : 0 signifie que $100 \%$ des répondants auraient donné à $100 \%$ des questions une réponse intolérante, 100, qu'à $100 \%$ des questions ils ont donné une réponse tolérante.

Les Français dans leur globalité ont en effet un rapport moins monocolore qu'il n'y paraît à l'immigration. Ainsi les principales peurs éprouvées par la société française sont moins centrées sur l'immigration et la diversité qu'il n'y paraît. En janvier 2016, deux mois après le Bataclan, le chômage reste la principale crainte de 22 \% des Français interrogés dans le baromètre de la $\mathrm{CNCDH}$, devant le terrorisme (18\%), la crise économique $(10,5 \%)$ ou la pauvreté $(9 \%)$. Les craintes identitaires restent finalement assez marginales: les personnes enquêtées ne sont que $6 \%$ à 
mettre en avant l'intégrisme religieux, $5 \%$ la "perte de l'identité de la France ", 4,5\% l'immigration. D'ailleurs ces enjeux ne mobilisent qu'à la droite de la droite et particulièrement chez les proches du FN, dont on sait qu'en cas de deuxième tour, ils se rabattent désormais essentiellement sur la droite plutôt que la gauche.

Autre fait marquant, contrairement à certains discours alarmistes, les Français sont restés relativement ouverts à l'immigration et à la diversité, notamment après les attentats de 2015. C'est ce que nous apprend l'indice longitudinal de tolérance ${ }^{7}$ qui synthétise l'ensemble des résultats du baromètre de la CNCDH depuis 1990. L'élection de François Hollande s'insère dans une chute brusque de l'indice jusqu'en 2013. Mais depuis lors, l'indice a retrouvé un niveau proche de celui de 2009. Ceci montre que les attentats ne produisent pas obligatoirement de la peur et du repli chez les Français.

Dans les dernières décennies, la France a connu les attentats islamistes de l'été 1995 à Paris, ceux de Washington et New York en septembre 2011, ceux de Madrid en mars 2004, ceux de Londres en juillet 2005 et ceux de L'Hyper Casher et de Charlie Hebdo en 2015. Pourtant, en 1995, 2001 et 2004 on ne constate pas de crispation raciste. Dans les deux premiers cas, l'indice reste stable et dans le troisième, la tolérance progresse même. Surtout, en 2015, entre la vague du baromètre de novembre 2014 et celle de février 2015, puis entre février 2015 et janvier 2016, les Français sont même devenus plus tolérants, notamment parmi l'électorat LR. Ceci met en avant le rôle essentiel des élites politiques dans la mise en récit et la compréhension des événements. Autrement dit, un discours différent sur l'immigration, qui fait la part belle aux apports de la diversité à notre société, est possible.

Cela se retrouve dans les séries de questions spécifiques de l'indice de tolérance : ainsi en 1992, seuls 44,5 \% des Français considéraient que l'immigration était une source d'enrichissement culturel ; ils sont 68,5\% en 2016. En 1992, 66 \% estimaient que " les travailleurs immigrés doivent être considérés ici comme chez eux puisqu'ils contribuent à l'économie française "; ils sont 79 \% en 2016. Enfin, ils n'étaient en 1997 que 47 \% à s'opposer à l'idée que " l'immigration est la source principale de l'insécurité "; ils sont encore $55 \%$ en 2016, malgré le contexte tendu actuel. Tous les préjugés n'ont pas disparu mais la dynamique va vers plus de tolérance et d'acceptation de la différence. Surtout elle est portée par deux phénomènes importants et cumulatifs.

D'abord par le renouvellement générationnel : ainsi les cohortes les plus âgées, qui sont également les plus fermées, se voient remplacées par des jeunes qui s'avèrent plus ouverts à la diversité. Ensuite par un effet
7. Pour connaître le mode de construction de cet indice voir : N. Mayer, G. Michelat, V. Tiberj, T. Vitale, "Le regard des chercheurs sur les différentes formes de préjugés ", in $\mathrm{CNCDH}$, La lutte contre le racisme et la xénophobie. 2015, Paris, La Documentation Française, p. 281-337, 2016.

\section{Deux mois après le Bataclan, le chômage reste la principale crainte de $22 \%$ des Français, (...) les attentas ne produisent pas obligatoirement de la peur et du repli.}


de période : la France vieillit certes, mais à année de naissance égale, les préjugés décroissent avec le temps. Autrement dit, les Français nés dans les années 1940 ont moins de préjugés aujourd'hui qu'en 1990. Enfin, par l'élévation du niveau de diplôme : en 1968, 45 \% des adultes n'ont aucun diplôme ; ils ne sont plus que $20 \%$ en 2006 ; en 1968, seuls $11 \%$ des Français détiennent un baccalauréat ou plus, ils sont $37 \%$ en 2006.

Bref, il y a une place et une dynamique pour parler autrement de l'immigration et de la diversité, ce d'autant plus que notre modèle d'intégration n'en est plus vraiment un.

\section{- La France, un modèle d'intégration ?}

Souvent la France s'érige en modèle, et c'est particulièrement le cas pour la question de l'intégration. Ainsi la tradition de jus solis est réguliè-

\section{La France n'est plus en avance en matière d'intégration et mériterait de se pencher sur les bonnes pratiques de ses voisins.} rement mise en avant face au jus sanguinis caractéristique de l'Allemagne (qui a considérablement assoupli sa position depuis 2000). Les gouvernements de gauche et de droite se prévalent régulièrement de cet héritage pour contrer les critiques que leur politique

8. G. NOIRIEL, Le creuset français, Paris, Seuil, 1992.

9. G NoIriel, Le massacre des Italiens : Aigues-Mortes, 17 aôut 1893, Paris, Fayard, 2010 ; P. Milza, Voyages en Ritalie, Paris, Plon, 1993.

10. http://www.mipex. $\mathrm{eu} /$ d'immigration (ou leur absence de politique volontariste) a suscité. Difficile de nier en effet que la République a accueilli les vagues migratoires depuis plus longtemps que la plupart des états européens, et dans des proportions parfois équivalentes à celles constatées aux États-Unis ${ }^{8}$. Pourtant la France n'est plus en avance et mériterait de se pencher sur les bonnes pratiques de ses voisins. Là aussi, la chancelière Merkel a fait un choix courageux, quand la gauche a tergiversé avant d'accueillir quelques milliers de réfugiés syriens.

Dans le débat public, les immigrés aujourd'hui sont souvent comparés aux immigrés des vagues précédentes, réputés plus prompts à vouloir s'intégrer dans la société hexagonale. C'est oublier combien ceux-ci ont été également confrontés à la xénophobie des "Français de souche". Il suffit pour s'en convaincre de se pencher sur la littérature historique9. Être polonais au début du siècle dans les mines lorraines, être italien dans les quartiers populaires de Marseille, être juif dans le marais parisien, provoquait un rejet qui n'est sans doute pas très différent de celui que subissent les immigrés et leurs enfants aujourd'hui. En 1991, 27 \% des personnes interrogées considéraient encore qu'il y avait trop d'immigrés originaires du sud de l'Europe en France ( $35 \%$ parmi les plus de 65 ans). Les préjugés à l'encontre du " rital " ou du " portos " ont survécu jusqu'à très récemment.

On dispose désormais de moyens de comparer les performances des différents États européens en matière de politiques d'intégration. C'est ainsi qu'a été créé le projet Mipex (Migrant integration policy index ${ }^{10}$ ) qui vise à identifier et comparer les bonnes pratiques des différents États sur 140 indicateurs de politiques publiques regroupés en six grandes dimensions : l'accès au marché du travail, le regroupement familial, la résidence 
de longue durée, la participation politique, l'accès à la nationalité du pays d'accueil et les législations anti-discriminations. La dernière livraison date de 2015, à un moment où les gouvernements socialistes avaient largement eu l'occasion de faire une différence avec la droite au pouvoir entre 2002 et 2012. La France est loin d'être le pays le plus favorable aux immigrés et à leurs droits, puisqu'elle se place en $18 \mathrm{e}$ position sur 38 pays étudiés. En

"Peu de choses ont changé dans la politique d'intégration française par rapport au gouvernement conservateur précédent. " fait, elle est même classée comme " moyenne" dans cinq des six grandes dimensions, avec des politiques considérées comme particulièrement rétrogrades dans de nombreux cas : en matière de reconnaissance des diplômes universitaires, de regroupement familial (où la France est désormais en queue de peloton notamment avec l'édiction des critères de solvabilité ou d'intégration), ou encore de sécurisation des permis de long-terme.

Bien sûr, certaines de nos politiques d'intégration sont encore considérées comme avancées: il en va ainsi des libertés politiques ou de la double nationalité. Mais la France, en comparaison avec ses homologues européens, n'est plus un exemple à suivre au regard des standards édictés par les instances de l'Union européenne, à la différence notamment de la Suède ${ }^{11}$. Surtout, les commentaires de l'indice sont particulièrement clairs quant au bilan de la gauche : Peu de choses ont changé dans la politique d'intégration française par rapport au gouvernement conservateur précédent, [...] le nouveau gouvernement a encore à tenir ses promesses de développer l'aide à l'apprentissage du français pour tous les nouveaux venus, de lutter contre les discriminations à l'école, de promouvoir le droit de vote des étrangers aux élections locales, de mettre en place un permis pluriannuel de séjour, une procédure plus claire vers la résidence permanente ${ }^{12}$ et une procédure moins discrétionnaire d'accès à la naturalisation".

Et encore, les créateurs du Mipex ne parlent pas des contrôles au faciès dont on sait combien ils tendent les relations entre police et population. Dans leur enquête, Fabien Jobard et ses collègues ${ }^{13}$ montrent combien indépendamment de sa tenue, de son âge ou de son sexe un passant d'apparence noir ou maghrébin aura largement plus de chances d'être contrôlé qu'un passant "blanc ". On sait que ces contrôles ont une incidence non négligeable sur la confiance que les Français d'origine immigrée ont à l'égard de la police ${ }^{14}$. L'abandon par le pouvoir socialiste du simple récépissé de contrôle, qui fait partie des routines d'actions de la police britannique depuis 1986, montre combien les débats français manquent encore une fois de pragmatisme.

\section{- La République inachevée}

Il faut sortir de nos frontières pour comprendre que d'autres pays, confrontés aux mêmes phénomènes d'immigration réagissent différemment

11. La montée de l'extrême droite aux dernières législatives pourrait cependant remettre en question ce modèle.

12. La loi de 2016 a finalement instauré une carte de séjour pour une durée maximale de quatre ans.

\section{F. JOBARD,}

R. LÉVY, J. LAMBerth, S. NÉVANEN, "Mesurer les discriminations selon l'apparence: une analyse des contrôles d'identité à Paris ", Population 3/2012 (Vol. 67), p. 423-451.

14. V. Tiberj, P. Simon, "La fabrique du citoyen. Origines et rapport au politique en France ", dans Beauchemin, C. Hamel, P. Simon (dir.), Trajectoires et origines : enquête sur la diversité des populations en France, Paris, INED, p. 501-530, 2016. 
et parfois mieux. On peut toujours arguer par exemple que la Belgique un peu avant la France a voté une loi prohibant la burqa. Mais elle donne le droit de vote aux étrangers non-européens, qui paraît désormais bien compromis en France, alors même que François Hollande a disposé d'une fenêtre d'opportunité particulièrement favorable: jamais les Français y avaient été aussi favorables (59\% en avril 2012) et jamais un Président socialiste n'avait eu autant de marges de manœuvre institutionnelles.

Au-delà même des politiques publiques à mener, c'est avant tout à un changement de notre manière de penser la République qu'il faut s'atteler. Trop souvent les questions relatives à l'immigration se heurtent à l'argument du modèle républicain. Le droit de vote des étrangers? En opposition avec notre conception de la citoyenneté. Les menus sans porc dans les cantines ou les fast-foods ? En opposition avec la laïcité républicaine, alors même que nombre de municipalités ont une approche très pragma-

\section{Au-delà même des politiques publiques à mener, c'est avant tout à un changement de notre manière de penser la République qu'il faut s'atteler.} tique et normalisée de ces questions. La discrimination positive? En opposition avec la méritocratie républicaine. La mesure des origines? En contradiction avec le principe de non-distinction des Français. Mais est-ce si républicain de dénier à des immigrés installés en France depuis plusieurs décennies le droit de participer à la désignation de certains des représentants du peuple, surtout depuis que les ressortissants des pays de l'Union européenne en disposent? Mais est-ce si républicain de refuser de tenir compte des interdits alimentaires de certains alors même que le jour du poisson dans les cantines scolaires tombe bien souvent le vendredi ?

Mais est-ce si républicain d'accepter que dans les grandes écoles, les enfants des catégories supérieures trustent l'essentiel des places, au détriment des enfants d'ouvriers et d'employés, parfois, mais pas que, issus de l'immigration? Mais est-ce si républicain de refuser de se donner les moyens de mesurer ces discriminations au quotidien qui plombent la vie de nos concitoyens parce qu'ils ont un nom particulier, une couleur de peau qui n'est pas blanche ou qu'on leur assigne une religion, des coutumes, des préjugés au faciès ? La Constitution proclame l'égalité entre tous indépendamment des origines, des races, des opinions ou du sexe. Et pourtant, ce n'est pas en voulant biffer de la Constitution le mot "race " comme l'a proposé François Hollande qu'on abolira le racisme. De plus la Constitution n'est pas la société, - où les sociologues ne cessent de constater ces inégalités dues à l'origine. Le déni de république n'est pas dans les mesures des origines, il est dans les discriminations. Où en serions-nous sans certains des résultats exposés ici, qui n'existent que parce qu'on a posé la question des origines?

Revenons un peu sur ce modèle républicain qu'on croit en danger. S'il est en danger, c'est qu'à un moment donné, il a connu un âge d'or, 
celui où il s'incarnait pleinement et qu'il faudrait retrouver. Essayons d'en trouver la date. La troisième République? Une période où les femmes n'avaient pas le droit de vote, où les indigènes avaient un statut bien à part, où l'administration coloniale comptait et classait entre Européens, métis et autochtones. La cinquième République ? Celle qui a attendu la fin des années 1960 pour que les femmes puissent ouvrir un compte en banque sans l'aval de leurs maris? Celle qui a voté la loi de 1969 qui oblige les Tsiganes à justifier de trois ans de résidence dans une commune pour être inscrit sur les listes électorales (contre six mois pour les autres citoyens français)? Cette loi n'a été déclarée incons-

\section{Le déni de république n'est pas dans les mesures des origines, il est dans les discriminations.} titutionnelle qu'à l'automne 2012.

La République n'a jamais été parfaite. Elle s'est toujours caractérisée par un équilibre entre des progrès et des grandes lois, mais aussi les défauts de l'époque qui ont été progressivement gommés. Notre République est une république inachevée, un objectif toujours à réaliser plutôt qu'une citadelle assiégée. 